\title{
Artigo/Article
}

\section{Análise da fonte alimentar de Panstrongylus megistus (Hemiptera, Reduviidae, Triatominae) e sua atual importância como vetor do Trypanosoma cruzi, no Estado de Minas Gerais}

\author{
Analysis on the food source of Panstrongylus megistus (Hemiptera, Reduviidae, Triatominae) \\ and its present importance as a vector for Trypanosoma cruzi, in the State of Minas Gerais
}

Marcos Marreiro Villela ${ }^{1,3}$, Vera Lúcia Cortiço Corrêa Rodrigues ${ }^{2}$, Cláudio Casanova $^{2}$ e João Carlos Pinto Dias ${ }^{1}$

\begin{abstract}
RESUMO
Introdução: A pesquisa da fonte alimentar dos triatomíneos auxilia no conhecimento sobre a biologia destes insetos, inclusive para inferir-se a importância dos mesmos como transmissores do Trypanosoma cruzi ao homem. Métodos: O presente estudo objetivou registrar, através da reação de precipitina, a fonte alimentar de triatomíneos provenientes de 54 municípios da região centro-oeste de Minas Gerais, assim como a infecção natural destes para flagelados semelhantes ao Trypanosoma cruzi. Resultados: De julho de 2003 até março de 2007, foram avaliados 416 insetos, todos da espécie Panstrongylus megistus. Somando-se as ocorrências, as aves (70\%) e os humanos $(22,5 \%)$ constituíram as principais fontes sanguíneas. Encontrou-se $16(3,8 \%)$ insetos positivos para flagelados semelhantes ao Trypanosoma cruzi sendo que oito realizaram hematofagia no homem. Conclusões: Os resultados demonstram necessidade de acentuar a vigilância entomológica na região, visto que foi detectada a possibilidade de ocorrência de novos casos da doença de Chagas através da transmissão vetorial.
\end{abstract}

Palavras-chaves: Doença de Chagas. Fonte alimentar. Panstrongylus megistus.

\begin{abstract}
Introduction: Investigation of the food source of triatomines helps in understanding the biology of these insects, and also helps to infer their importance in transmitting Trypanosoma cruzi to humans. Methods: The present study aimed to use the precipitin reaction to record the food source of triatomines from 54 municipalities in the central-western region of the State of Minas Gerais, as well as their natural infection by flagellates similar to Trypanosoma cruzi. Results: From July 2003 to March 2007, 416 insects were evaluated, all from the species Panstrongylus megistus. Among all these occurrences, birds (70\%) and humans (22.5\%) were the major blood sources. Sixteen (3.8\%) of the insects were found to be positive for flagellates similar to Trypanosoma cruzi, and eight performed hematophagia on humans. Conclusions: The results show the need to increase the entomological surveillance in this region, given that the possibility of new cases of Chagas disease occurring through vector transmission was detected.
\end{abstract}

Key-words: Chagas disease. Food source. Panstrongylus megistus.

1. Laboratório de Triatomíneos, Centro de Pesquisas René Rachou, Fundação Oswaldo Cruz, Belo Horizonte, MG. 2. Laboratório de Referência da Doença de Chagas, Superintendência do Controle de Endemias, Mogi Guaçu, SP. 3. Instituto de Biologia, Universidade Federal de Pelotas. Pelotas, RS.

Endereço para correspondência: Dr. Marcos Marreiro Villela. Rua General Osório 1213, 96020-000 Pelotas, RS.

Tel: 5553 3275-7620.

e-mail: marcos.villela@ufpel.edu.br

Recebido para publicação em 07/07/2009

Aceito em 01/12/2009

\section{INTRODUÇÃO}

A doença de Chagas ainda figura como grave problema de saúde pública no Brasile naAmérica Latina ${ }^{1}$. Os vetores desta enfermidade são insetos hematófagos da subfamília Triatominae (Hemíptera: Reduviidae), composta atualmente de 141 espécies $^{2}$. Em Minas Gerais são capturadas 15 espécies de triatomíneos ${ }^{3}$, entretanto, após a eliminação de Triatoma insfestans, espécie introduzida no estado no final do século XIX, Panstrongylus megistus voltou a ser a espécie mais capturada na região. Existe uma acentuada tendência de domiciliação desta espécie em várias regiões do Brasil, principalmente no Centro, Leste e Sudeste do país e, provavelmente, as matas residuais constituem os focos de abrigo para as populações deste triatomíneo ${ }^{4,5}$.

Em Minas Gerais, Panstrongylus megistus tem sido frequentemente capturado no ambiente domiciliar, e sua colonização ocorre principalmente no peridomicílio, onde as aves domésticas representam importante fonte de alimentação, atuando como mantenedoras de colônias de triatomíneos ${ }^{6}$.

O estudo da fonte alimentar de triatomíneos é realizado com frequência, dada a importância que as informações daí provenientes contribuem para ampliar o conhecimento de seus hospedeiros naturais e seu papel na transmissão do Trypanosoma cruzi ao homem 7 . Várias técnicas podem ser utilizadas com esta finalidade, desde a análise microscópica do tipo de hemácia ingerida, até técnicas de biologia molecular8 ${ }^{8}$. Em 1960, um trabalho realizado por Siqueira mostrou ser possível obter, através da reação de precipitina, a origem alimentar de triatomíneos, mesmo daqueles que haviam se alimentado há mais de 120 dias?.

Já foi demonstrado que Panstrongylus megistus apresenta marcante ecletismo alimentar, constatandose que sua alimentação é realizada em aves, humanos, cães, gatos, marsupiais, roedores, bovinos, caprinos, suínos, dentre outros ${ }^{10}$. Um estudo sobre Panstrongylus megistus realizado em Bambuí, região centro-oeste 
de Minas Gerais, demonstrou relevante participação do homem como fonte alimentar deste triatomíneo, uma vez que em $40 \%$ dos insetos identificou-se sangue humano no seu conteúdo intestinal, demonstrando a possibilidade de transmissão do Trypanosoma cruzi por este vetor ${ }^{11}$.

O objetivo desta pesquisa foi identificar as fontes alimentares de espécimes de Panstrongylus megistus coletados em diversos municípios da região centro-oeste de Minas Gerais, em vista da constância com que a espécie tem sido capturada em domicílios da região ${ }^{12}$.

\section{MÉTODOS}

Elegeu-se para o estudo a região centro-oeste de Minas Gerais, constituída por 54 municípios, os quais são coordenados administrativamente pela Gerência Regional de Saúde (GRS) de Divinópolis, quanto ao Programa de Controle da Doença de Chagas (PCDCh) e de outras endemias. Tal região trata-se de um dos locais onde a enfermidade foi mais frequente, existindo, na mesma, importante cunho histórico sobre a enfermidade e seu controle ${ }^{13}$. Além deste fato, importa salientar que a região tem sido alvo de estudos sobre o aperfeiçoamento da vigilância e controle da doença de Chagas, nos últimos anos ${ }^{12,14,15}$.

Os triatomíneos foram coletados pelos moradores e agentes municipais do PCDCh, sendo, posteriormente, encaminhados aos laboratórios da GRS Divinópolis e do Centro de Pesquisas René Rachou, Fundação Oswaldo Cruz (Belo Horizonte, MG). O conteúdo do tubo digestivo dos insetos capturados foi preparado no laboratório da GRS Divinópolis, sendo armazenados em lâminas de papel filtro Whatman número 3, e enviadas para a FIOCRUZ de Belo Horizonte (MG). Nesta instituição, os dados foram digitalizados e as amostras enviadas para a Superintendência do Controle de Endemias (SUCEN) de Mogi Guaçu/São Paulo, na qual foram secas e estocadas a $-20^{\circ} \mathrm{C}$ para identificação da fonte alimentar através da reação de precipitina ${ }^{9}$. As coletas foram feitas entre julho de 2003 até junho de 2007. Os antissoros adotados na reação contemplam os prováveis hospedeiros que ocorrem na área de estudo, sendo compostos por: homem, felinos, caninos, marsupiais, roedores, suínos e aves.

\section{RESULTADOS}

De julho de 2003 até março de 2007, foi analisado o conteúdo intestinal de 460 insetos, provindos de 110 localidades de 29 municípios, todos pertencentes à espécie Panstrongylus megistus. Destes, 44 amostras não reagiram aos tipos de antissoro testados ou o material foi considerado insuficiente. As aves constituíram a principal fonte alimentar dosinsetos, seguido do sangue de humanos (Tabela 1). Dos 416 insetos que reagiram, $376(90,4 \%)$ tinham ingerido sangue de uma única origem, e 40 (9,6\%) alimentaram-se de duas ou mais fontes. A associação mais frequente, considerando-se todas as amostras, foi sangue de ave com sangue humano (4,6\%). Cumpre ressaltar que 141 $(33,9 \%)$ triatomíneos provieram do intradomicílio e 275 (66,1\%) foram colhidos no peridomicílio (Tabela $\mathbf{1}$ ).

Agrupando-se os insetos que se alimentaram de uma fonte, com os que se alimentaram de duas ou mais, o sangue de aves foi detectado em 291 (70\%) amostras, seguido de sangue humano, encontrado no tubo digestório de 94 (22,5\%) insetos (Figura 1).

Dos triatomíneos analisados, $16(3,8 \%)$ estavam positivos para flagelados semelhantes ao Trypanosoma cruzi. Destes, quatro insetos se alimentaram de duas fontes. Todavia, somando-se as
TABELA 1 - Fonte alimentar de Panstrongylus megistus capturados na região centro-oeste de Minas Gerais, entre julho de 2003 e junho de 2007.

\begin{tabular}{lcc}
\hline Fonte alimentar & Número & Porcentagem \\
\hline Ave & 263 & 63,2 \\
Humano & 68 & 16,4 \\
Gambá & 17 & 4,1 \\
Gato & 13 & 3,2 \\
Cão & 9 & 2,2 \\
Roedor & 3 & 0,7 \\
Porco & 3 & 0,7 \\
Ave + humano & 19 & 4,6 \\
Humano + gambá & 6 & 1,5 \\
Ave + gambá & 3 & 0,7 \\
Ave + gato & 2 & 0,5 \\
Gambá + gato & 2 & 0,5 \\
Cão + gato & 2 & 0,5 \\
Humano + roedor & 1 & 0,2 \\
Gambá + cão & 1 & 0,2 \\
Ave + cão & 1 & 0,2 \\
Ave + roedor + cão & 1 & 0,2 \\
Ave + gambá + cão & 1 & 0,2 \\
Ave + cão + gato & 1 & 0,2 \\
\hline Total & $\mathbf{1 0 0 , 0}$ \\
\hline
\end{tabular}

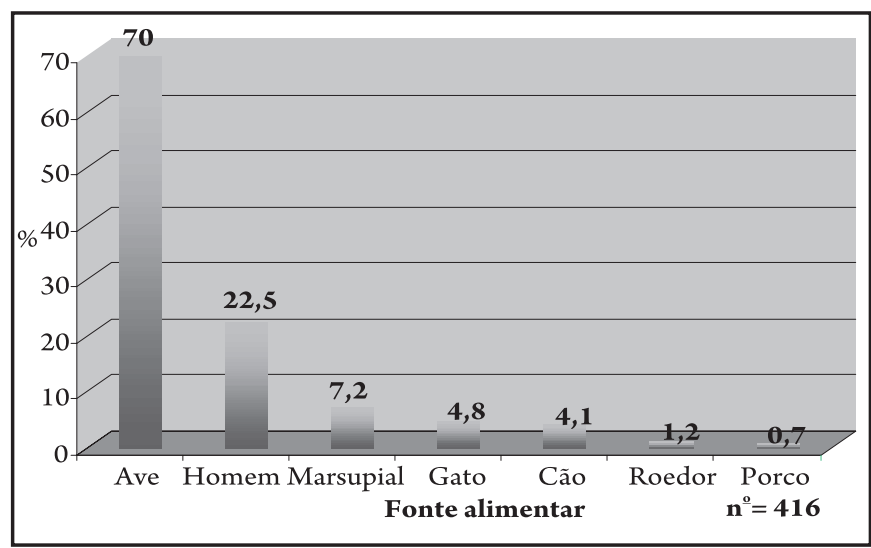

FIGURA 1 - Tipo de sangue ingerido por triatomíneos capturados em municípios da região centro-oeste de Minas Gerais, de acordo com a reação de precipitina, entre julho de 2003 e junho de 2007.

ocorrências, oito triatomíneos realizaram hematofagia no homem, sete alimentaram-se em aves, dois em cães e dois em gatos.

Ressalta-se que, nas residências onde se encontraram insetos positivos, foram realizados exames sorológicos dos moradores para se diagnosticar novos casos da doença de Chagas (serviço prestado pelas prefeituras e supervisionado pela GRS Divinópolis); entretanto, nenhum caso novo da moléstia foi diagnosticado. Um senhor acusou sorologia positiva para Trypanosoma cruzi, contudo o paciente já havia sido previamente diagnosticado (Figura 1).

Quanto à localização dosinsetos, 80,4\% dos que se alimentaram de aves foram capturados no peridomicílio. Ressalta-se que 10,6\% dos triatomíneos alimentados com sangue humano se encontravam no peridomicílio, e $19,6 \%$ dos que ingeriram sangue de ave se encontravam no intradomicílio (Tabela 2). Portanto, a proporção intradomicílio/peridomicílio para resultados de fonte humana e de ave foi, respectivamente, de 89,4\%/10,6\% e 19,6\%/80,4\%. Existe correlação significativa entre a fonte alimentar do inseto e o local onde o mesmo é capturado $(\mathrm{p}<0,05)$ (Tabela 2). 
TABELA 2 - Local de captura dos triatomíneos coletados na região centrooeste de Minas Gerais, de acordo com a fonte alimentar, entre julho de 2003 e junho de 2007.

\begin{tabular}{|c|c|c|c|c|c|c|}
\hline \multirow[b]{2}{*}{ Fonte alimentar } & \multicolumn{2}{|c|}{ Intradomicílio } & \multicolumn{2}{|c|}{ Peridomicílio } & \multicolumn{2}{|c|}{ Total } \\
\hline & $\mathrm{n}^{\mathrm{o}}$ & $\%$ & $\mathrm{n}^{\mathrm{o}}$ & $\%$ & $\mathrm{n}^{\mathrm{o}}$ & $\%$ \\
\hline Ave & 57 & 19,6 & 234 & 80,4 & 291 & 100,0 \\
\hline Humano & 84 & 89,4 & 10 & 10,6 & 94 & 100,0 \\
\hline Marsupial & 10 & 33,3 & 20 & 66,7 & 30 & 100,0 \\
\hline Gato & 4 & 20,0 & 16 & 80,0 & 20 & 100,0 \\
\hline Cão & 4 & 23,5 & 13 & 76,5 & 17 & 100,0 \\
\hline Roedor & 2 & 40,0 & 3 & 60,0 & 5 & 100,0 \\
\hline Porco & - & 0,0 & 3 & 100,0 & 3 & 100,0 \\
\hline
\end{tabular}

\section{DISCUSSÃO}

Na atual pesquisa, identificou-se ecletismo alimentar de Panstrongylus megistus, o qual se alimentou de pelo menos sete fontes alimentares distintas, concordando com dados da literatura ${ }^{10,16,17}$. A ocorrência de resultados não reagentes da precipitina é, possivelmente, oriunda da insuficiência de material sanguíneo na amostra do conteúdo intestinal, ou à possibilidade de o animal que serviu de fonte alimentar não estar contemplado na bateria de antissoros utilizada.

As aves foram a principal fonte alimentar diagnosticada no presente estudo (70\%), fato que pode ser explicado pelo maior número de capturas no peridomicílio, sobretudo no galinheiro, onde se deram $66,1 \%$ destas capturas. Importante destacar que alguns desses hemípteros podem ter chegado ao ambiente artificial já ingurgitados de sangue de aves silvestres. Os insetos positivos para sangue de aves já eram esperados considerando a frequência com que galinhas vivem próximas ao homem e frequentemente servirem de fonte alimentar para triatomíneos ${ }^{18}$. Achado semelhante já ocorreu na mesma região, e os autores chegaram a um índice próximo de Panstrongylus megistus reagentes para sangue de aves através da reação de precipitina $(74,6 \%)$ no município de Bambui ${ }^{11}$. Inúmeras outras espécies, como Triatoma infestans e, mais comumente, Triatoma sordida, também podem apresentar as aves como principal fonte alimentar, na dependência de sua detecção em ecótopos habitados por estes animais ${ }^{19}$.

A presença de insetos no peridomicílio que apresentam sangue humano no seu tubo digestório, sugere que alguns triatomíneos realizaram migrações ativas entre o intra e o peridomicílio, em duplo sentido, tanto insetos adultos como as ninfas (raros casos). Por seu turno, a presença de $19,6 \%$ dos insetos ingurgitados com sangue de aves e capturados no intradomicílio, reforça o conceito de que os focos peridomiciliares constituem importante espaço de reserva e manutenção de populações triatomínicas ${ }^{20,21}$. Estudos epidemiológicos apontam que as aves domésticas apresentam papel importante na dinâmica de transmissão do Trypanosoma cruzi ao homem, visto que embora refratárias, servem como fonte de alimentação para os triatomíneos no ambiente peridomiciliar, permitindo a presença de populações abundantes destes vetores próximas do homem, afora servirem de elo entre o ambiente silvestre e o ambiente domiciliar ${ }^{22}$.

$\mathrm{O}$ alto índice de triatomíneos que se alimentaram de sangue humano $(22,5 \%)$ denota o significativo grau de antropofilia de Panstrongylus megistus, o que já foi demonstrado em outras avaliações na região ${ }^{11,21}$. Os achados atuais demonstraram maior antropofilia de Panstrongylus megisus do que os dados compilados no Estado de São Paulo para esta mesma espécie, onde o percentual de insetos ingurgitados por sangue humano ficou em $9 \%$. Isso possivelmente se dê em decorrência da maior proporção de insetos capturados no intradomicílio no presente estudo quando comparado aos dados de São Paulo, onde 91,5\% dos insetos foram achados no âmbito peridomiciliar ${ }^{23}$.

A preferência por sangue humano é um dos motivos que intervém na capacidade vetorial de um triatomíneo ${ }^{24-26}$. Quando comparado a outras espécies, especialmente com Triatoma sordida, Panstrongylus megistus normalmente apresenta maiores índices de antropofilia, mostrando elevado ecletismo alimentar, capacidade de domiciliação e mobilidade espacial, com menores graus de ornitofilia ${ }^{5,8,27}$.

Segundo Martins ${ }^{28}$, à existência da infecção chagásica humana sob a forma endêmica se faz possível onde existirem triatomíneos domiciliados com fácil acesso ao homem, que lhes fornece alimentação sanguínea e a quem os vetores podem transmitir o protozoário Trypanosoma cruzi. Nesta avaliação, a presença de insetos positivos para flagelados semelhantes ao Trypanosoma cruzi que ingeriram sangue humano, mostra claramente a possibilidade de transmissão vetorial que ocorre na região. Por sorte, a transmissão de Trypanosoma cruzi a um mamífero é um evento que ocorre com baixas probabilidades, em virtude de que a forma de contaminação através do vetor é pouco eficiente se comparado às vias de transmissão que ocorrem para outros patógenos (inoculação do microorganismo através do aparato picadorsugador do vetor). Devido a este fator, a probabilidade de transmissão é baixa, e o número de contatos do triatomíneo com o homem precisa ser abundante para que a transmissão ocorra ${ }^{22}$. Outra característica que deve ser considerada é que além da possível transmissão do Trypanosoma cruzi para o homem e mamíferos domésticos, tais vetores ocasionam incômodo e irritação durante o repouso das pessoas e realizam espoliação sanguínea, podendo contribuir para doenças alérgicas e mesmo para anemia por deficiência de ferro ${ }^{29}$.

No presente estudo, também, foram encontradas outras fontes alimentares dos triatomíneos, entre as quais se destacam os marsupiais $(7,2 \%)$, cães $(4,8 \%)$, gatos $(4,1 \%)$ e roedores $(1,2 \%)$. No que tange os marsupiais, os resultados foram abaixo do esperado, visto que Panstrongylus megistus é o hemíptero mais comumente associado aos marsupiais, vertebrados que se encontram corriqueiramente contaminados pelo Trypanosoma cruzi ${ }^{16,30,31}$. Talvez este seja um dos fatores para explicar a frequência relativamente baixa de infecção dos insetos capturados nesta observação, quando comparado aos estudos citados anteriormente: a diminuta taxa de insetos ingurgitados por sangue de marsupiais.

Com relação aos roedores, a proporção de insetos que se alimentaram dos mesmos obteve índices inferiores a um estudo realizado no Estado de São Paulo, também analisando-se Panstrongylus megistus, entretanto os insetos capturados provinham do meio silvestre, onde mais comumente encontram-se associados a roedores ${ }^{2}$. No Estado do Paraná, uma pesquisa revelou que 35,3\% dos insetos (Panstrongylus megistus) avaliados foram reativos para sangue de roedores, além de também se encontrarem reações positivas para sangue de cão e de aves, e embora a amostra pesquisada tenha sido reduzida, aponta o ecletismo alimentar da espécie ${ }^{33}$. Numa última análise, todos estes mamíferos (gambás, roedores, cães e gatos) apresentam papel importante na transmissão do Trypanosoma cruzi, visto que os mesmos, quando infectados, costumam manter alta taxa de infecção para os triatomíneos devido à elevada parasitemia que habitualmente apresentam ${ }^{22}$. Complementando a afirmação anterior, outras pesquisas também demonstraram que a presença de cães e gatos influencia no aumento da infecção dos triatomíneos, sendo importantes no ciclo de transmissão da moléstia no ambiente doméstico ${ }^{34-36}$. 
Concluí-se que Panstrongylus megistus continua a ter importância como espécie vetora do Trypanosoma cruzi ao homem na região, sendo encontrado no domicílio e peridomicílio, nos quais se depara com abrigo adequado e abundante de fonte alimentar. Embora as taxas de incidência e prevalência da $\mathrm{DCH}$ por meio da transmissão vetorial sigam diminuindo no Brasil ${ }^{37,38}$ a presença de Panstrongylus megistus ingurgitado de sangue humano e, em alguns casos, positivos para flagelados semelhantes ao Trypanosoma cruzi, demonstra real possibilidade de novas ocorrências da doença de Chagas na região centro-oeste de Minas Gerais, reforçando a necessidade de manutenção e aprimoramento da vigilância nos municípios em questão.

\section{CONFLITO DE INTERESSE}

Os autores declaram não haver nenhum tipo de conflito de interesse no desenvolvimento do estudo.

\section{REFERÊNCIAS}

1. Uchôa E, Firmo JA, Dias EC, Pereira MSN, Gontijo EDG. Signos, significados e ações associados à doença de Chagas. Cad Saúde Pública 2002; 18:71-79.

2. Diotaiuti L, Oliveira MA (orgs) Triatomíneos. Centro de Pesquisas René Rachou, Fundação Oswaldo Cruz, Belo Horizonte, 2009.

3. Lent H, Wigodzinsky P. Revision of the triatominae (Hemíptera: Reduviidae), and their significance as vectors of Chagas disease. Bull Am Mus Nat Hist 1979; 163: 123-520.

4. Forattini OP. Biogeografia, origem e distribuição da domiciliação de triatomíneos no Brasil. Rev Saúde Pública 1980; 14: 265-299.

5. Silva RA, Wanderley DMV, Domingos MF, Yasumaro S, Scandar SAS, PauliquévisJúnior C, et al. Doença de Chagas: notificação de triatomíneos no Estado de São Paulo na década de 1990. Rev Soc Bras Med Trop 2006; 39: 488-494.

6. Villela MM. Vigilância entomológica da doença de Chagas na região centro-oeste de Minas Gerais, Brasil. Tese de Doutorado. Centro de Pesquisas René Rachou/ Fundação Oswaldo Cruz, Belo Horizonte, MG; 2009.

7. Forattini OP, Barata JMS, Santos JLF, Silveira AC. Hábitos alimentares, infecção natural e distribuição de triatomíneos domiciliados na região central do Brasil. Rev Saúde Pública São Paulo 1982; 16:171-204.

8. Lorosa ES, Andrade RE, Pereira CA, Santos SM, Juberg J. Identificação das fontes alimentares de Triatoma sordida (Stal, 1859), (Hemíptera-Reduviidae) na região norte de Goiás, Brasil, através da técnica de precipitina. Entomologia y Vectores 1999; 6: 291-304.

9. Siqueira AF. Estudo sobre a reação da precipitina aplicada a identificação de sangue ingerido por triatomíneos. Rev Inst Med Trop São Paulo 1960; 2:41-53.

10. Carcavallo RU, Rocha DS, Girón I, Sherlock IA, Galvão C, Martinez A. Fontes e padrões alimentares. In: Carcavallo RU, Girón GI, Juberg J, Lent H (orgs) Atlas dos vetores da doença de Chagas nas Américas. Rio de Janeiro: Editora da Fundação Oswaldo Cruz; 1997. p. 537-560.

11. Fernandes AJ, ChiariE, Casanova C,DiasJCP,Romanhã AJ. The threat of reintroduction of natural transmission of Chagas disease in Bambuí, Minas Gerais State, Brazil, due to Panstrongylus megistus. Mem Inst Oswaldo Cruz 1992; 87: 285-289.

12. Villela MM, Souza JMB, Melo VP, Dias JCP. Avaliação do Programa de Controle da doença de Chagas em relação à presença de Panstrongylus megistus na região centrooeste do Estado de Minas Gerais, Brasil. Cad Saúde Pública 2009; 25:907-917.

13. Dias JCP, Prata A, Coura JR. Emmanuel Dias: o principal artífice do combate à doença de Chagas nas Américas. Rev Soc Bras Med Trop 2008; 41: 502-504.

14. Villela MM, Souza JB, Mello VP, Azeredo BVM, Dias JCP. Vigilância entomológica da doença de Chagas na região centro-oeste de Minas Gerais, Brasil, entre os anos de 2000 e 2003. Cad Saúde Pública 2005; 21: 878-886.

15. Villela MM, Souza JMB, Melo VP, Dias JCP. Vigilância epidemiológica da doença de Chagas em programa descentralizado: avaliação de conhecimentos e práticas de agentes municipalizados em região endêmica de Minas Gerais, Brasil. Cad Saúde Pública 2007; 23:2428-2438.

16. Barretto MP, Siqueira AF, Freitas JP. Estudos sobre reservatórios e vectores silvestres do Trypanosoma cruzi. II- Encontro de Panstrongylus megistus em ecótopos silvestres no estado de São Paulo (Hemiptera, Reduviidae). Rev Inst Med Trop São Paulo 1964; 6: 56-63.

17. Barretto MP. Estudos sobre reservatórios e vectores silvestres do Trypanosoma cruzi. XXXI - Observações sobre a associação entre reservatórios e vectores, com especial referência a região Nordeste do Estado de São Paulo. Rev Bras Biol 1968; 28: 481-494.

18. Freitas JLP, Siqueira AF, Ferreira OA. Investigações epidemiológicas sobre triatomíneos de hábitos domésticos e silvestres com auxílio da reação de precipitina. Rev Inst Med Trop São Paulo 1960; 2: 90-99.

19. Gajate PP, Bottazz MV, Pietrokovsky SM, Wisnivesky-ColF C. Relação entre Triatoma infestans, aves domésticas e o Homem num povoado de Santiago del Estero, Argentina. Cad Saúde Pública 1994; 10: 53-57.

20. Dias JCP. Participação, descentralização e controle de endemias no Brasil. In Barata RB, Briceño-León R (eds) Doenças Endêmicas, Rio de Janeiro: Editora da Fundação Oswaldo Cruz; 2000. p. 269-297.

21. Diotaiuti L. Ocorrência e biologia de Rhodnius neglectus Lent, 1954, em macaubeiras da periferia de Belo Horizonte, MG. Dissertação de Mestrado, Universidade Federal de Minas Gerais, MG; 1982.

22. Gorla DE. Que características podem definir espécies/populações de triatomíneos com maior potencial vetorial? Rev Soc Bras Med Trop 2001; 34: 81-82.

23. Rodrigues VLCC, Casanova C, Baitelo D, Mattos CN, Tonietti VLB. Aspectos relacionados com a infecção por Trypanosoma cruzi em Panstrongylus megistus (Burmeister, 1835), capturados na região de Campinas, Estado de São Paulo, Brasil. Rev Soc Bras Med Trop 2006; 39:156.

24. Barretto MP. Epidemiologia. In: Brener Z, Andrade Z (eds)rypanosoma cruzi e doença de Chagas. Rio de Janeiro: Guanabara Koogan; 1979. p. 89-151.

25. Dias JCP, Coura JR. Epidemiologia. In: Dias JCP, Coura JR (orgs) Clínica e terapêutica da doença de Chagas. Rio de Janeiro: Editora Fundação Oswaldo Cruz; 1997. p. 33-65.

26. Zeledón R, Rabinovich JE. Chagas disease: An ecological appraisal with special emphasis on its Insect Vectors. Annu Rev Entomol 1981; 26:101-133.

27. Lorosa ES, Andrade RE, Pereira CA, Santos SM, Jurberg J. Estudo das fontes alimentares Triatoma sordida (Stal, 1859) do Estado do Mato Grosso do Sul através da comparação das técnicas de precipitina e imunodifusão dupla. Entomologia y Vectores 1999; 6: 156-165.

28. Martins AV. Epidemiologia da doença de Chagas. In: Cançado JR. Doença de Chagas. Belo Horizonte: Imprensa Oficial; 1968. p. 225-237.

29. Schofield CJ, Maudlin I. Trypanosomiasis control. Int J Parasitol 2001; 31: 615-620.

30. Forattini OP, Rabello EX, Castanho MLS, Pattoli DGB. Aspectos ecológicos da triapanossomose americana. I- Observações sobre Panstrongylus megistus e suas relações com focos naturais de infecção, em área urbana da cidade de São Paulo. Rev Saúde Pública 1970; 4: 19-30.

31. Miles MA, Souza AAA, Povoa MM. O ecótopo de Panstrongylus megistus (Hemíptera, Reduviidae) na floresta do horto (Rio de Janeiro). Rev Bras Biol 1982; 42: 31-35.

32. Barretto MP, Siqueira AF, Ferriolli Filho F, Carvalheiro JR. Estudos sobre reservatórios e vetores naturais do Trypanosoma cruzi. XX: Infecção natural de ratos comensais capturados em biótopos naturais e artificiais, por tripanossomos semelhantes ao T. cruzi. Rev Bras Biol 1967; 2: 145-156.

33. Falavigna-Guilherme AL, Lorosa ES, Costa AL, Pavanelli GC, Araújo SM Panstrongylus megistus em ecótopos artificiais de ilhas do Alto Rio Paraná. Rev Soc Bras Med Trop 2001; 34: 491-494.

34. Catalá S, Crocco L, Morales G, Paulone I, Giraldez E, Candiotti C. Trypanosoma cruzi transmission risk index: a tool to improve vector control. Annual Report. Geneva: World Health Organization (Tropical Disease Research); 1996.

35. Gürtler RE, Wisniveski-Colli C, Solarz ND, Lauricella M, Bujas MA. Dynamics of transmission of Trypanosoma cruzi in a rural area of Argentina: II. Household infection patterns among children and dogs relative to the density of infected Triatoma infestans. Bull Pan Am Health Organ 1987; 21: 280-292.

36. Silva RP. Estudo sobre Trypanosoma (Schizotrypanum) cruzi Chagas, 1909, em área de Mato Grosso do Sul: casos humanos, reservatórios e transmissores. Tese de Doutorado. Universidade Estadual de Mato Grosso do Sul, Campo Grande, MS; 1979.

37. Miles MA, Yeo M, Gaunt MW. Epidemiology of American Trypanosomiasis. In: Maudlin I, Holmes PH, Miles MA (orgs) The Trypanosomes. London: CABI Publishing; 2004. p. 243-251.

38. World Health Organization. Control of Chagas Disease. Geneva: World Health Organization; 2002. 